\title{
Diversité Floristique Des Lianes De La Forêt Classée De Bouaflé, Centre-Ouest De La Côte D'ivoire
}

\section{Dro Bernadin,}

Ufr Agroforesterie, Université Jean Lorougnon Guede, Daloa, Côte D'ivoire, Centre Suisse De Recherches Scientifiques En Côte D'ivoire, Abidjan, Côte

D'ivoire

N'goran Koffi Désiré,

Coulibaly Siendou,

Salla Moreto,

Amon Anoh Denis-Esdras,

Kouassi Kouadio Henri,

Ufr Agroforesterie, Université Jean Lorougnon Guede, Daloa, Côte D’ivoire

Doi:10.19044/esj.2020.v16n27p17～URL:http://dx.doi.org/10.19044/esj.2020.v16n27p17

\section{Résumé}

Les lianes, une des composantes importantes de la flore des forêts tropicales sont bien souvent négligées dans des travaux d'étude floristique. La diversité de ces plantes dans quatre types d'occupation du sol (TOS) de la forêt classée de Bouaflé a été étudiée. Il s'agit de trois types de reboisement (Teck, association Fraké-Framiré, hévéa) et des jachères. Les inventaires floristiques utilisant la méthode de surface ont été réalisés dans des parcelles d'un hectare $(100 \mathrm{~m} \times 100 \mathrm{~m})$. Ainsi, 522 individus de 45 espèces de lianes appartenant à 39 genres et 20 familles ont été inventoriés. Les TOS jachères et plantations de Fraké-Framiré ont été les plus riches et diversifiées en lianes tandis que ceux de Teck et d'hévéa ont été les plus pauvres et moins diversifiées. Dans l'ensemble des TOS, les familles les plus importantes ont été les Passifloraceae (VIF $=63,97)$, les Fabaceae $(\mathrm{VIF}=52,08)$, les Caesalpiniaceae $(\mathrm{VIF}=39,25)$ et les Combretaceae $(\mathrm{VIF}=27,90)$. Adenia lobata $(\mathrm{IVI}=50,33)$, Acacia pennata $(\mathrm{IVI}=28,41)$ et Combretum zenkeri $(\mathrm{IVI}=27,15)$ ont été les espèces les plus importantes. L'analyse de la structure diamétrique a montré une population stable de lianes. Cette étude a montré que, pour une reconstitution efficiente du couvert végétal, les reboisements plurispécifiques sont préférables aux reboisements monospécifiques.

Mots clés : Diversité floristique, Lianes, Types d'occupation du sol, Forêt classée, Côte d'Ivoire 


\title{
Floristic Diversity Of Lianas In Bouaflé Classified Forest, Central West Côte D'ivoire
}

\section{Dro Bernadin,}

Ufr Agroforesterie, Université Jean Lorougnon Guede, Daloa, Côte D'ivoire, Centre Suisse De Recherches Scientifiques En Côte D'ivoire, Abidjan, Côte

D'ivoire

N'goran Koffi Désiré,

Coulibaly Siendou,

Salla Moreto,

Amon Anoh Denis-Esdras,

Kouassi Kouadio Henri,

Ufr Agroforesterie, Université Jean Lorougnon Guede, Daloa, Côte D’ivoire

\begin{abstract}
Lianas, this important component of tropical forest flora are mostly neglected in several studies. The objective of this study was to evaluate the floristic diversity of these plants in four types of land use (Teak, Shinglewoodblack Afara association, hevea, Fallows) in Bouaflé Classified Forest. Lianas at $1 \mathrm{~cm} \mathrm{DBH}$ were inventoried in sampling blocks of 1 hectare $(100 \mathrm{~m} \times 100$ $\mathrm{m}$ ) divided in eight elementary blocks of $25 \mathrm{~m}$ x 20. Results showed that 522 lianas included in 45 species, 39 genera and 20 families were recorded. In addition, Fallows and Shinglewood-black Afara association were the richest and most diversified in lianas while Teak and Hevea plantations were the poorest and less diversified. Regarding the Family Importance Value, the most important families were Passifloraceae $(\mathrm{VIF}=63,97)$, Fabaceae $(\mathrm{VIF}=$ 52,08), Caesalpiniaceae (VIF $=39,25)$ and Combretaceae $(\mathrm{VIF}=27,90)$. According to Importance value Index (IVI), Adenia lobata (IVI $=50,33$ ), Combretum zenckeri $(\mathrm{IVI}=27,15)$ and Acacia pennata $(\mathrm{IVI}=28,41)$ were the most represented species in all type of land use. Furthermore, the distribution in diameter classes showed a stable population of lianas. This study showed that multispecifical reforestation plans are preferable to that of single species.
\end{abstract}

Keywords: Floristic diversity, Lianas, Types of land use, Classified forest, Côte d'Ivoire 


\section{Introduction}

Les forêts tropicales se caractérisent, non seulement, par la grande diversité des espèces arborescentes mais aussi, par l'abondance des espèces lianescentes (Alemo, 2014). Les lianes constituent ainsi une composante essentielle de la flore de ces milieux. En effet, la flore lianesente représente, environ, $25 \%$ des espèces ligneuses et, en moyenne, 18\% de la diversité taxonomique (Ewango, 2010).

L'importance des lianes est indéniable et perceptible à divers niveaux. Ces plantes jouent souvent le rôle de pont, d'échelle. Près de la canopée dans les étages supérieurs de la forêt, elles permettent à de nombreuses formes d'espèces animales de parcourir la hauteur des arbres sans parfois jamais descendre au sol (Schnitzer et Bonger, 2002). Aussi, elles jouent un rôle déterminant dans la dynamique forestière. À ce titre, elles limitent la régénération d'arbres, augmentent leur mortalité, fournissent une source alimentaire vitale aux animaux et lient physiquement les végétaux. Ainsi, elles contribuent fortement à l'équilibre de la biodiversité et à l'épanouissement de la faune.

Par ailleurs, les lianes ont un intérêt non négligeable dans la vie des populations humaines et animales. Certaines espèces sont reconnues pour leurs vertus thérapeutiques et effets bénéfiques sur la santé humaine (Eilu et Bukenya-Zirabab, 2004). C'est le cas, par exemple, de Morinda morindoides (Baker) Milne-Redh. (Rubiaceae) utilisé dans le traitement du paludisme et de Abrus precatorius L. (Fabaceae) reconnue pour son potentiel antivenimeux rapporté contre les morsures de serpents (Tra Bi, 2002).

Malheureusement, ces espèces de valeur sont, comme toutes les autres composantes de la flore forestière, sous la menace de la pression anthropique. En effet, de vastes superficies de forêts sont détruites pour, entre autres, l'exploitation forestière, les activités agricoles et les grands travaux de développement (routes, construction, urbanisation, etc.).

En Côte d'Ivoire, par exemple, la forêt qui occupait toute la moitié Sud du pays, a souffert d'une dégradation accélérée à partir des années 1970 (environs 12 millions d'hectares en 1970). À ce jour, la couverture forestière ivoirienne est estimée à seulement 2,7 millions d'hectares (FAO, 2005) avec un taux de déforestation annuel estimé à 4,32\% entre 1990 et 2005. La composition floristique des forêts se trouve ainsi fortement modifiée. Au cours de ces transformations, les lianes ne sont pas épargnées. Certaines risquent même de disparaitre avec tous leurs intérêts avant même d'être étudiées. Koffi et $a l$, (2016) soutiennent que, dans le processus de reconstitution naturelle d'une végétation forestière, les lianes sont les premières espèces à coloniser l'espace.

Devant cette situation alarmante de réduction drastique de sa biodiversité, la Côte d'Ivoire a créé des aires protégées dont la forêt classée de Bouaflé dans le but de préserver la diversité biologique. La gestion de cette forêt classée 
comme celle du genre est confiée à la Société de Développement des Forêts (SODEFOR). La réussite du plan de gestion et d'aménagement de ce milieu passe par la connaissance de sa biodiversité. Or à notre connaissance, très peu de travaux de recherche ont été menés dans la forêt classée de Bouaflé. Les études connues sur ce milieu sont de Yao (2017) et de Sidibé (2015) et ont porté respectivement sur les types de reboisement et l'impact de la pression anthropique. Il n'y a jusqu'à ce jour pas eu d'étude consacrée aux lianes de la forêt classée de Bouaflé.

Cette étude a été réalisée pour connaître la diversité des lianes dans la forêt classée de Bouaflé. Elle est une contribution à la mise en place des données scientifiques sur ces plantes de valeur en vue d'une meilleure gestion.

\section{Méthodologie}

\section{Site d'étude}

Les travaux ont été conduits dans la Forêt Classée de Bouaflé (FCB) située dans la région de Daloa (Figure 1), au Centre-Ouest de la Côte d'Ivoire. D'une superficie de 20350 hectares, la FCB est située près de la localité de Daloa entre $6^{\circ} 46^{\prime}$ et $6^{\circ} 55^{\prime}$ de latitude Nord et $6^{\circ} 04^{\prime}$ et $6^{\circ} 15^{\prime}$ de longitude Ouest (SODEFOR, 2014). Elle bénéficie des mêmes conditions climatiques que cette localité. Le climat est de type subéquatorial Attiéen (Adjiri et al., 2020) comportant deux saisons à savoir une saison sèche et une saison des pluies. La saison sèche s'étend sur quatre mois (novembre à février). La saison des pluies dure huit mois et s'étend de mars à octobre.

La pluviométrie est élevée entre Mars et Octobre et faible entre Novembre et Février. La pluviométrie moyenne est très faible en janvier (environs $10 \mathrm{~mm}$ de pluie chaque année). Les mois les plus humides sont avril, août et septembre. Ils enregistrent, en moyenne, $103,58 \mathrm{~mm}$ de pluies chaque année.

La température moyenne annuelle est de $26,3^{\circ} \mathrm{C}$. Les mois de novembre et mai sont les plus secs avec respectivement les températures moyennes de 26,2 et $27,9{ }^{\circ} \mathrm{C}$. 


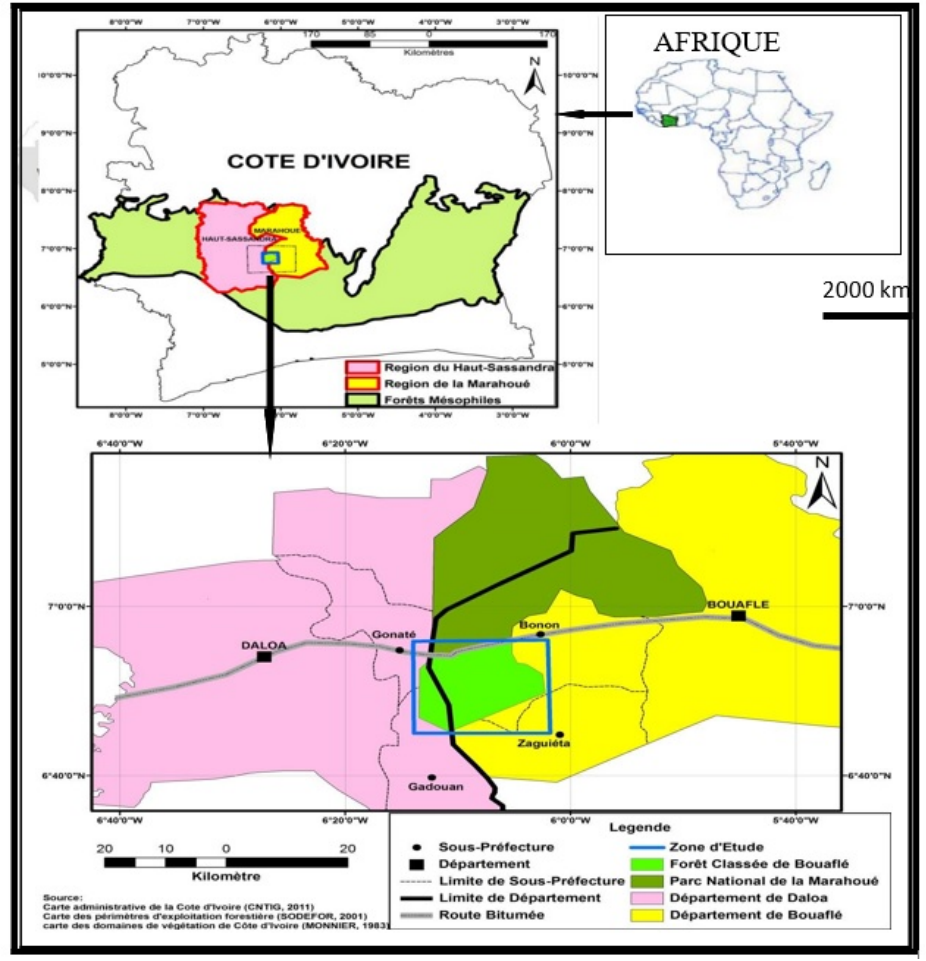

Figure 1: Carte de la forêt classée de Bouaflé (Sidibé, 2015 modifiée)

\section{Collecte des données}

\section{Mise en place des placettes d'inventaire des lianes}

Les inventaires floristiques ont été réalisés dans quatre types d'occupation du sol de la forêt classée de Bouaflé. Il s'agit des reboisements de Tectona grandis L. f. (Verbenaceae), des reboisements associant Terminalia superba Engl. \& Diels (Combretaceae) et Terminalia ivorensis A. Chev (Combretaceae), des reboisements de Hevea brasiliensis (A. Juss.) Müll. Arg. (Euphorbiaceae) et des jachères.

La méthode de relevés de surface basée sur la mise en place des placettes a été utilisée. C'est une méthode classique déjà utilisée par Koubouana et al. (2018) et Gnahoré et al. (2020). Ainsi, des placettes d'un hectare, soit $100 \mathrm{~m}$ x $100 \mathrm{~m}$, ont été délimitées dans chaque TOS. Chaque placette a, ensuite, été subdivisée en 25 sous-parcelles carrées de $400 \mathrm{~m}^{2}$ ( $20 \mathrm{~m}$ x $20 \mathrm{~m}$ ) à l'aide du ruban métrique de $30 \mathrm{~m}$.

\section{Inventaire floristique des lianes}

L'inventaire floristique a été réalisé dans cinq sous-parcelles. Il s'agit des quatre sous-parcelles angulaires et de la sous-parcelle centrale. À l'intérieur de chacune d'elles, toutes les lianes rencontrées ont été identifiées et inventoriées. Chaque individu inventorié a été marqué soit à la machette soit 
à l'encre indélébile afin de ne pas être prise en compte plus d'une fois. La circonférence de tous les individus de lianes rencontrées a été mesurée à l'aide d'un ruban métrique de 1,5 $\mathrm{m}$ de longueur sans aucune condition et portée sur la fiche de relevé floristique conçue à cet effet.

Des échantillons d'espèces de lianes non identifiées sur le terrain ont été collectés dans des sacs plastiques pour identification au laboratoire de Biologie végétale de l'Université Jean Lorougnon Guédé de Daloa. Le référentiel taxonomique pour l'Afrique tropicale de Lebrun et Stork (19912015) et la flore de l'Afrique de l'Ouest de Bongers et al. (2005) ont servi de guide pour les identifications. Le système de nomenclature adopté est celui de Cronquist (1960).

\section{Traitement des données}

Deux principaux paramètres régulièrement utilisés pour évaluer la prépondérance spécifique dans les forêts tropicales (Dro, 2014) ont été utilisés. Il s'agit de l'Indice de Valeur d'Importance (IVI) de Cottam et Curtis (1956) et de l'indice de Valeur d'Importance des Familles (VIF) de Mori et al. (1983).

\section{Évaluation de l'importance relative des lianes}

L'Indice de Valeur d'Importance (IVI) caractérise l'importance, au sein d'une végétation, d'une espèce par rapport à l'ensemble des autres espèces (Gueulou et al., 2018). L'importance relative est définie comme étant la somme de la densité relative (DR), de la fréquence relative (FR) et de la dominance relative de chaque espèce (Dro, 2014). La formule de l'Indice de Valeur d'Importance d'une espèce est :

$$
\begin{aligned}
& \text { IVI }=\text { DR + FR + DoR } \\
& \text { Avec : } \\
& \text { - } \mathrm{DR}=\frac{\text { Nombre } \mathrm{d}^{\prime} \text { individus de l'espèce }}{\text { Somme totale des individus de toutes les espèces }} \times 100 \\
& \text { FR }=\frac{\text { Nombre de sous placettes où l'espèce est apparue } \times 100}{\text { Somme totale des nombres de sous placettes d'apparition de toutes les espèces }} \\
& \text { DoR }=\frac{\text { Aire basale de l'espèce }}{\text { Somme totale des aires basales de toutes les espèces }} \times 100
\end{aligned}
$$

L'aire basale ou surface terrière d'une espèce se définit comme la surface occupée par la section des troncs de ses individus à hauteur de poitrine ou à $1,30 \mathrm{~m}$ au-dessus du sol.

Aire basale $=(\mathrm{C} / 2 \pi)^{2} \times \pi$, où $\mathrm{C}=$ circonférence $(\mathrm{m})$ et $\pi=22 / 7$

Ainsi, l'IVI permet de distinguer les espèces les plus abondantes, fréquentes, dominantes et finalement les espèces les plus importantes dans le milieu (Dro, 2014). Dans cette étude, le diamètre des lianes inventoriées étant 
trop petit, une espèce a donc été considérée comme écologiquement importante lorsque son IVI $\geq 25$. Les espèces ayant un IVI $\leq 25$, désignées par autres, sont considérées comme moins importantes dans la FCB.

\section{Appréciation de la Valeur d'Importance des Familles de lianes}

L'indice de Valeur d'Importance des Familles (VIF) de Mori et al. (1983) a été calculé pour apprécier les familles de liane prépondérantes dans l'ensemble de la FCB et dans chaque TOS. L'indice de VIF est la somme de trois facteurs quantitatifs représentatifs. Il s'agit de la densité relative (DR), de la diversité relative (DiR) et de la dominance relative (DoR). La formule de l'indice de Valeur d'Importance des Familles, pour une famille donnée, est :

$$
\begin{gathered}
\mathbf{V I F}=\mathbf{D R}+\mathbf{D i R}+\mathbf{D o R} \\
\text { Avec : } \\
\text { DR }=\frac{\text { Nombre d'individus de la famille }}{\text { Nombre total de tous les individus inventoriés }} \times 100 \\
\text { DiR }=\frac{\text { Nombre d'espèces de la famille }}{\text { Nombre total d'espèces }} \times 100 \\
-\quad \text { DoR }=\frac{\text { Somme des aires basales des individus de la famille }}{\text { Somme totale des aires basales des individus de toutes les familles }} \times 100
\end{gathered}
$$

Une famille a été considérée comme abondante lorsque VIF $\geq 25$.

D'autres paramètres ont été utilisés pour caractériser les lianes de cette forêt. Il s'agit du coefficient de similitude de Sørensen (1948) et de la structure diamétrique.

\section{Similarité entre les types d'occupation du sol étudiés}

Le coefficient de similitude de Sørensen (1948) a été utilisé pour évaluer la ressemblance floristique entre les TOS à partir de leurs listes floristiques. Pour deux milieux A et B, il est calculé comme suit :

$$
\mathrm{Cs}=\frac{2 \mathrm{C}}{\mathrm{A}+\mathrm{B}} \times 100
$$

où Cs : le coefficient de similitude de Sørensen, C : le nombre d'espèces communes aux deux milieux, A : le nombre d'espèces du milieu $\mathrm{A}, \mathrm{B}$ : le nombre d'espèces du milieu $B$.

Les valeurs de Cs varient entre 0 et $100 \%$. Plus les milieux ont des espèces communes, plus Cs tend vers $100 \%$. Dans le cas contraire, le Cs tend vers 0. Les milieux dont la valeur du Cs est supérieure à $50 \%$ présentent une ressemblance floristique ; ceux dont le Cs est inférieur à $50 \%$ présentent une dissimilarité floristique. 


\section{Appréciation de la structure diamétrique des lianes}

La répartition des individus de liane par classe de diamètres a été établie afin d'apprécier les classes d'âges (Dro, 2014), c'est-à-dire les individus ayant un petit ou un gros diamètre. Ainsi, six classes de diamètres ont été retenues dans ce travail : $[0 ; 1[,[1 ; 2[,[2 ; 3[,[3 ; 4[,[4 ; 5[$ et $\geq 5 \mathrm{~cm}$.

\section{Résultats}

\section{Richesse floristique en lianes de la Forêt Classée de Bouaflé}

Au total, 522 individus de lianes, appartenant à 20 familles, 39 genres et 45 espèces, ont été recensés. Les familles les plus représentées, en termes d'individus, sont celles des Passifloraceae et des Fabaceae (Figure 2) avec respectivement 91 et 88 individus. Ces deux familles totalisent 179 individus soit 34,29\% des individus dénombrés. Les familles minoritaires sont les Dilleniaceae et les Vitaceae. Elles ne sont représentées que par deux individus chacune soit $0,76 \%$.

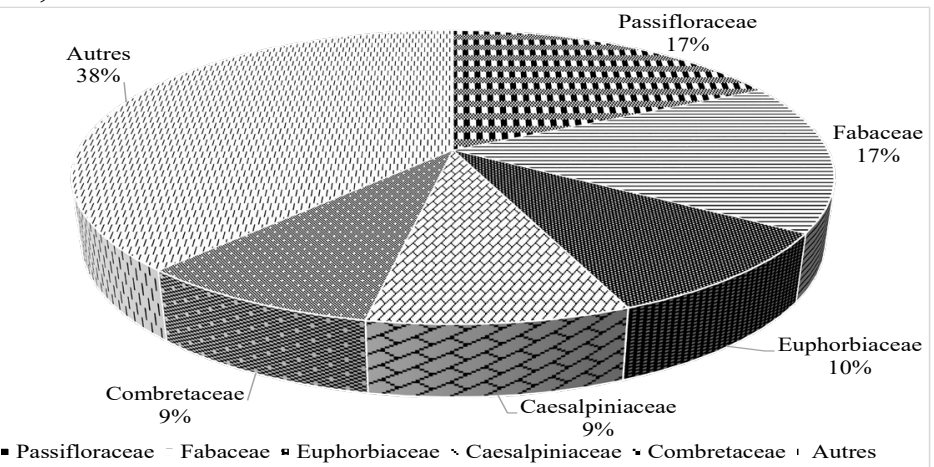

Figure 2: Proportion des familles les plus représentées dans l'ensemble des types d'occupation du sol de la Forêt Classée de Bouaflé

Trois genres sont majoritairement représentés en termes d'individus. Ce sont Adenia (73 individus), Combretum (46 individus) et Acacia (35 individus). Vingt genres renferment moins de 10 individus chacun et représentent 18,19\% des individus inventoriés. Seul le genre Loeseneriella est représenté par un seul individu.

Les jachères et les plantations de Fraké-Framiré sont les TOS les plus riches avec respectivement 223 et 172 individus. Les plantations d'hévéa sont les plus pauvres avec 49 individus. La richesse floristique en lianes des différents TOS est présentée dans le Tableau 1.

Tableau 1: Richesse floristique en lianes des types d'occupation du sol de la forêt classée de Bouaflé

\begin{tabular}{ccccc}
\hline $\begin{array}{c}\text { Types } \\
\text { d'occupation } \\
\text { du sol }\end{array}$ & $\begin{array}{c}\text { Nombre } \\
\text { d'individus }\end{array}$ & $\begin{array}{c}\text { Nombre } \\
\text { d'espèces }\end{array}$ & $\begin{array}{c}\text { Nombre de } \\
\text { genres }\end{array}$ & $\begin{array}{c}\text { Nombre de } \\
\text { familles }\end{array}$ \\
\hline Teck & 78 & 17 & 16 & 13 \\
\hline
\end{tabular}




\begin{tabular}{ccccc} 
Fraké-Framiré & 172 & 27 & 26 & 16 \\
\hline Jachères & 223 & 35 & 32 & 17 \\
\hline Hévéa & 49 & 16 & 15 & 11 \\
\hline
\end{tabular}

En considérant la composition floristique, les TOS les plus riches en familles, en genres et en espèces sont les jachères, suivies des plantations de Fraké-Framiré et de Teck. Les plantations d'hévéa sont les plus pauvres en liane.

La Figure 3 montre la proportion des familles les plus représentées dans chaque TOS de la forêt classée de Bouaflé. Les familles les plus représentées dans les plantations de Teck sont les Hippocrateaceae, les Combretaceae et les Fabaceae avec respectivement 22, 15 et 12 individus pour une proportion totale de l'ordre de $62,82 \%$ des individus de lianes inventoriés.

Dans les plantations de Fraké-Framiré, les familles les plus dominantes sont les Passifloraceae et les Caesalpiniaceae avec respectivement 39 et 30 individus.

Quant aux jachères, elles sont dominées par les Fabaceae avec 48 individus soit $21,52 \%$ des individus de lianes inventoriés, suivies des Passifloraceae (35 individus) et des Euphorbiaceae (32 individus).

La famille la plus représentée dans les plantations d'hévéa est celle des Passifloraceae avec 15 individus, soit 30,61\% des individus dénombrés. Elle est suivie des Fabaceae ( 7 individus) et des Periplocaceae (6 individus).
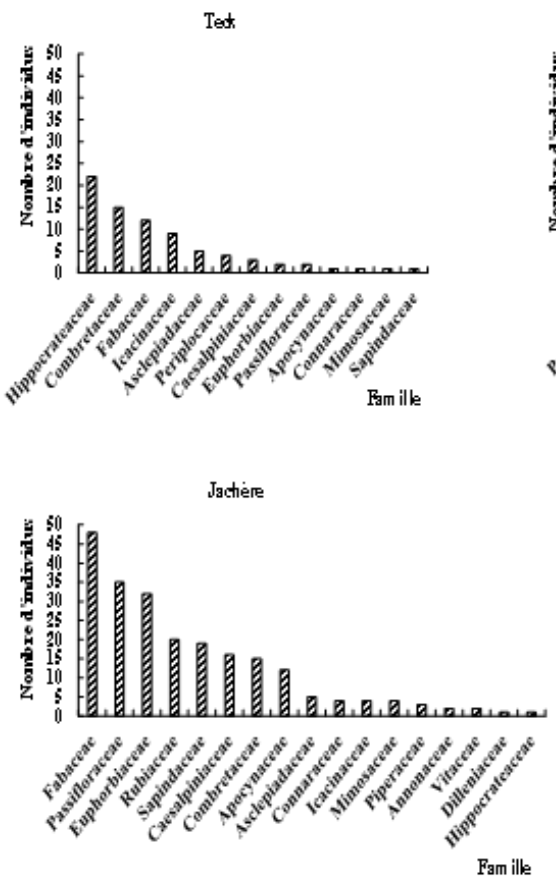

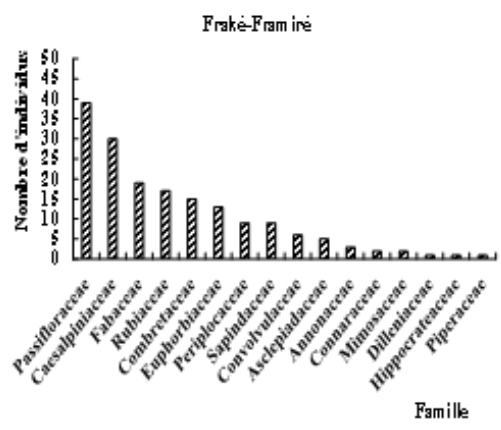

Hèca

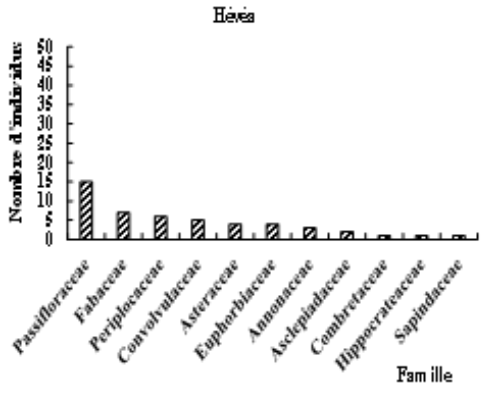

Figure 3: Proportion des familles de lianes prépondérantes dans les types d'occupation du sol de la Forêt Classée de Bouaflé 


\section{Importance relative des espèces}

Les espèces les plus importantes selon leur Indice de Valeur d'Importance (IVI) dans la FCB sont présentées dans le Tableau 2. Seules trois espèces sont importantes dans l'ensemble des TOS. Elles présentent chacune un IVI $\geq 25$. Ce sont Adenia lobata (Jacq.) Engl. (IVI = 50,33), Acacia pennata (L.) Willd $(\mathrm{IVI}=28,41)$ et Combretum zenkeri Engl. \& Diels (IVI = 27,15). Les autres 42 espèces ont chacune un IVI $<25$. En effet, Adenia lobata (Jacq.) Engl. est représentée par un grand nombre d'individus (espèce la plus abondante) avec de gros diamètres lui conférant une forte densité relative $(\mathrm{DR}=11,91 \%)$ et une forte dominance relative ( $\mathrm{DoR}=29,04 \%)$. Elle est également présente dans plusieurs placettes d'où sa forte fréquence relative $(\mathrm{Fr}=9,38)$. Quant à l'espèce Acacia pennata (L.) Willd, elle est représentée par des individus de gros diamètres $(\mathrm{DoR}=16,31 \%)$. Combretum zenkeri Engl. \& Diels est représentée aussi par un grand nombre d'individus $(\mathrm{DR}=8,83 \%)$ avec de gros diamètres ( $\mathrm{DoR}=12,59 \%)$.

Tableau 2: Espèces les plus importantes dans l'ensemble de la Forêt Classée de Bouaflé

\begin{tabular}{ccccc}
\hline Espèce & DR (\%) & FR (\%) & DoR (\%) & IVI \\
\hline Adenia lobata (Jacq.) Engl. & 11,91 & 9,38 & 29,04 & $\mathbf{5 0 , 3 3}$ \\
\hline Acacia pennata (L.) Willd & 6,37 & 5,73 & 16,31 & $\mathbf{2 8 , 4 1}$ \\
\hline Combretum zenkeri Engl. \& Diels & 8,83 & 5,73 & 12,59 & $\mathbf{2 7 , 1 5}$ \\
\hline Autres (40 espèces) & 72,9 & 79,2 & 42 & $\mathbf{1 9 4 , 1 1}$ \\
\hline
\end{tabular}

FR : Fréquence Relative ; DR : Densité Relative ; DoR : Dominance Relative ; IVI : Indice de Valeur d'Importance

Quant aux espèces importantes dans chaque TOS (Tableau 3), Adenia lobata (Jacq.) Engl. est importante dans trois TOS à savoir Fraké-Framiré, jachère et hévéa. Combretum zenkeri Engl. \& Diels est importante dans deux TOS (Teck et Fraké-Framiré) et Acacia pennata (L.) Willd présente uniquement dans les Jachères. En dehors de ces espèces communes, chaque TOS a ses propres espèces importantes.

Les espèces importantes dans les plantations de Teck sont au nombre de trois. Il s'agit des espèces Salacia owabiensis Hoyle, Combretum zenkeri Engl. \& Diels et Icacina mannii Oliv.. Dans les plantations de Fraké-Framiré, quatre espèces sont importantes dont Adenia lobata (Jacq.) Engl., Combretum zenkeri Engl. \& Diels, Griffonia simplicifolia (Vahl ex DC.) Bail et Rutidea smithii Hiern. Quant aux jachères, trois espèces sont importantes. Il s'agit des espèces Acacia pennata (L.) Willd, Adenia lobata (Jacq.) Engl. et Griffonia simplicifolia (Vahl ex DC.) Bail. Au niveau des plantations d'hévéa, les espèces importantes sont Adenia lobata (Jacq.) Engl., Cryptolepis calophylla (Baill.) L. Joubert \& Bruyns, Passiflora foetida Linn., Adenia cissampeloides (Planch. ex Hook.) Harms et Phyllanthus muellerianus (Kuntze) Exell. 


\section{Importance relative des familles}

Les familles les plus importantes, selon leur Valeur d'Importance des Familles (VIF) dans la FCB, sont présentées dans le Tableau 4. Seules quatre familles sont importantes (VIF $\geq 25$ ) dans l'ensemble des TOS. Ce sont les Passifloraceae (VIF $=63,97)$, les Fabaceae $(\mathrm{VIF}=52,08)$, les Caesalpiniaceae $(\mathrm{VIF}=39,25)$ et les Combretaceae $(\mathrm{VIF}=27,90)$. Les 16 autres familles ont chacune un VIF $<25$. En effet, les espèces appartenant à la famille des Passifloraceae $(\mathrm{DiR}=17,19 \%)$ et des Fabaceae $(\mathrm{DiR}=17,71 \%)$ sont présentes dans plusieurs placettes. Aussi, ces espèces appartenant à ces familles sont représentées par un grand nombre d'individus avec de gros diamètres. Ce qui confère aux Passifloraceae $(\mathrm{DR}=17,66 \%$; DoR $=29,12 \%)$ et aux Fabaceae $(\mathrm{DR}=16,63 \% ; \mathrm{DoR}=17,74 \%)$, une forte densité et dominance relatives. Quant aux Caesalpiniaceae et aux Combretaceae, les espèces ont de gros diamètres. Ce qui confère également aux Caesalpiniaceae (DoR $=29,99 \%)$ et aux Combretaceae $(\mathrm{DoR}=12,61 \%)$ une forte dominance relative.

Tableau 3: Espèces les plus importantes des types d'occupation du sol selon leur valeur d'importance

\begin{tabular}{|c|c|c|c|c|c|}
\hline TOS & Espèce & $\begin{array}{l}\text { DR } \\
(\%)\end{array}$ & $\begin{array}{l}\text { FR } \\
(\%)\end{array}$ & $\begin{array}{l}\text { DoR } \\
(\%)\end{array}$ & IVI \\
\hline \multirow{4}{*}{ Teck } & Salacia owabiensis Hoyle & 27,03 & 26,47 & 20,65 & 74,15 \\
\hline & Combretum zenkeri Engl. \& Diels & 17,57 & 8,82 & 41,56 & 67,95 \\
\hline & Icacina mannii Oliv. & 12,16 & 5,88 & 13,94 & 31,98 \\
\hline & Autres (14 espèces) & 43,24 & 58,82 & 23,85 & 125,92 \\
\hline \multirow{5}{*}{ Fraké-Framiré } & Adenia lobata (Jacq.) Engl. & 20,89 & 14,29 & 37,54 & 72,71 \\
\hline & Combretum zenkeri Engl. \& Diels & 9,49 & 7,14 & 16,57 & 33,21 \\
\hline & $\begin{array}{c}\text { Griffonia simplicifolia (Vahl ex DC.) } \\
\text { Bail }\end{array}$ & 9,49 & 5,36 & 17,02 & 31,87 \\
\hline & Rutidea smithii Hiern & 5,70 & 5,36 & 17,45 & 28,50 \\
\hline & Autres (23 espèces) & 54,43 & 67,86 & 11,42 & 133,71 \\
\hline \multirow{4}{*}{ Jachères } & Acacia pennata (L.) Willd & 12,74 & 11,25 & 24,44 & 48,43 \\
\hline & Adenia lobata (Jacq.) Engl. & 9,91 & 8,75 & 27,11 & 45,77 \\
\hline & $\begin{array}{l}\text { Griffonia simplicifolia (Vahl ex DC.) } \\
\text { Bail }\end{array}$ & 2,36 & 5,00 & 19,78 & 27,14 \\
\hline & Autres (31 Espèces) & 75,00 & 75,00 & 28,66 & 178,66 \\
\hline \multirow{5}{*}{ Hévéa } & Adenia lobata (Jacq.) Engl. & 4,65 & 3,70 & 28,96 & 37,32 \\
\hline & $\frac{\text { Cryptolepis calophylla (Baill.) L. Joubert }}{\underline{\text { \& Bruyns }}}$ & 13,95 & 11,11 & 11,91 & 36,97 \\
\hline & Passiflora foetida Linn. & 18,60 & 14,81 & 3,39 & 36,81 \\
\hline & $\begin{array}{c}\text { Adenia cissampeloides (Planch. ex } \\
\text { Hook.) Harms }\end{array}$ & 9,30 & 14,81 & 5,31 & 29,43 \\
\hline & Phyllanthus muellerianus (Kuntze) Exell & 6,98 & 7,41 & 10,91 & 25,29 \\
\hline
\end{tabular}




\section{Autres (10 espèces)}

46,51

48,15

39,51

134,17

TOS : Type d'occupation du sol ; FR : Fréquence Relative ; DR : Densité Relative; DoR : Dominance Relative; IVI : Indice de Valeur d'Importance

Tableau 4: Familles de lianes les plus importantes dans l'ensemble de la Forêt Classée de Bouaflé selon leur valeur d'importance

\begin{tabular}{ccccc}
\hline Famille & DR (\%) & DiR (\%) & DoR (\%) & VIF \\
\hline Passifloraceae & 17,66 & 17,19 & 29,12 & $\mathbf{6 3 , 9 7}$ \\
\hline Fabaceae & 16,63 & 17,71 & 17,74 & $\mathbf{5 2 , 0 8}$ \\
\hline Caesalpiniaceae & 9,45 & 7,81 & 21,99 & $\mathbf{3 9 , 2 5}$ \\
\hline Combretaceae & 9,03 & 6,25 & 12,61 & $\mathbf{2 7 , 9 0}$ \\
\hline Autres (16 familles) & 47,23 & 51,04 & 18,53 & $\mathbf{1 1 6 , 8 0}$ \\
\hline
\end{tabular}

DiR : Diversité Relative ; DR : Densité Relative ; DoR : Dominance Relative ; VIF : Valeur d'Importance des Familles

Quant aux familles importantes dans chaque TOS, elles sont présentées dans le Tableau 5. Les Passifloraceae et les Fabaceae sont importantes chacune dans trois TOS. Quant aux Caesalpiniaceae et Combretaceae, elles sont importantes chacune dans deux TOS.

Tableau 5: Familles de lianes les plus importantes des types d'occupation du sol de la Forêt Classée de Bouaflé

\begin{tabular}{|c|c|c|c|c|c|}
\hline TOS & Famille & DR (\%) & $\operatorname{DiR}(\%)$ & DoR (\%) & VIF \\
\hline \multirow{5}{*}{ Teck } & Hippocrateaceae & 27,03 & 26,47 & 20,65 & 74,15 \\
\hline & Combretaceae & 18,92 & 11,76 & 41,94 & 72,62 \\
\hline & Fabaceae & 14,86 & 14,71 & 4,85 & 34,42 \\
\hline & Icacinaceae & 12,16 & 5,88 & 13,94 & 31,98 \\
\hline & Autres (9 familles) & 27,03 & 41,18 & 18,62 & 86,83 \\
\hline \multirow{5}{*}{ Fraké-Framiré } & Passifloraceae & 23,42 & 17,86 & 37,61 & 78,89 \\
\hline & Caesalpiniaceae & 17,09 & 14,29 & 21,42 & 52,80 \\
\hline & Rubiaceae & 8,86 & 8,93 & 17,88 & 35,67 \\
\hline & Combretaceae & 9,49 & 7,14 & 16,57 & 33,21 \\
\hline & Autres (12 familles) & 41,14 & 51,79 & 6,51 & 99,44 \\
\hline \multirow{6}{*}{ Jachères } & Fabaceae & 21,70 & 20,00 & 24,89 & 66,59 \\
\hline & Passifloraceae & 15,57 & 15,00 & 27,17 & 57,74 \\
\hline & Caesalpiniaceae & 7,55 & 11,25 & 23,77 & 42,57 \\
\hline & Euphorbiaceae & 13,68 & 13,75 & 1,75 & 29,18 \\
\hline & Rubiaceae & 8,96 & 6,25 & 12,18 & 27,39 \\
\hline & Autres (11 familles) & 32,55 & 33,75 & 10,24 & 76,54 \\
\hline \multirow{5}{*}{ Hévéa } & Passifloraceae & 32,56 & 33,33 & 37,67 & 103,56 \\
\hline & Fabaceae & 16,28 & 14,81 & 11,98 & 43,08 \\
\hline & Periplocaceae & 13,95 & 11,11 & 11,91 & 36,97 \\
\hline & Euphorbiaceae & 6,98 & 7,41 & 10,91 & 25,29 \\
\hline & Autres (6 familles) & 30,23 & 33,33 & 27,53 & 91,10 \\
\hline
\end{tabular}

TOS : Type d'occupation du sol ; DiR : Diversité Relative, DR : Densité Relative, DoR :

Dominance Relative, VIF : Valeur d'Importance des Familles 


\section{Similarité floristique entre les types d'occupation du sol étudiés}

Le tableau 6 présente les coefficients de similitude entre les différents TOS pris deux à deux. Il ressort que les jachères et les plantations de Fraké-Framiré sont très semblables du point de vue de leurs compositions lianescentes, avec $71 \%$ de coefficient de similitude.

De même, les compositions lianescentes des jachères et des teckeraies $(53,80 \%)$, ainsi que celles des teckeraies et les plantations de Fraké-Framiré $(54,54 \%)$ sont semblables. Les autres couples sont floristiquement dissemblables.

Tableau 6: Matrice de similarité entre les différents TOS inventoriés dans la FCB

\begin{tabular}{ccccc}
\hline TOS & Teck & Fraké-Framiré & Jachère & Hévéa \\
\hline Teck & 1 & & & \\
\hline $\begin{array}{c}\text { Fraké- } \\
\text { Framiré }\end{array}$ & $54,54 \%$ & 1 & & \\
\hline Jachère & $53,80 \%$ & $71 \%$ & 1 & \\
\hline Hévéa & $42,42 \%$ & $41,86 \%$ & $35,29 \%$ & 1 \\
\hline \multicolumn{5}{c}{ TOS : Type d'occupation du sol }
\end{tabular}

\section{Structure diamétrique des lianes inventoriées}

La structure diamétrique des lianes dans l'ensemble des TOS inventoriés est en forme de J inversé (Figure 4). La FCB renferme plus de lianes de petits diamètres $(<1 \mathrm{~cm})$ que celles de gros diamètres. Le nombre d'individus de liane diminue à mesure que leur diamètre augmente. Ce constat est le même au niveau de chaque TOS (Figure 5). Ainsi, la structure diamétrique est en forme de $\mathrm{J}$ inversé dans tous les TOS.

Toutes les classes de diamètres sont représentées dans les plantations de Fraké-Framiré et dans les jachères. Par contre, aucun individu de diamètre $\geq 4$ $\mathrm{cm}$ n'a été inventorié dans les plantations de Teck. De plus, toutes les lianes des plantations d'hévéa sont de très petit diamètre $(<1 \mathrm{~cm})$. Les lianes de diamètre supérieur n'ont pas eu de représentant dans ce TOS.

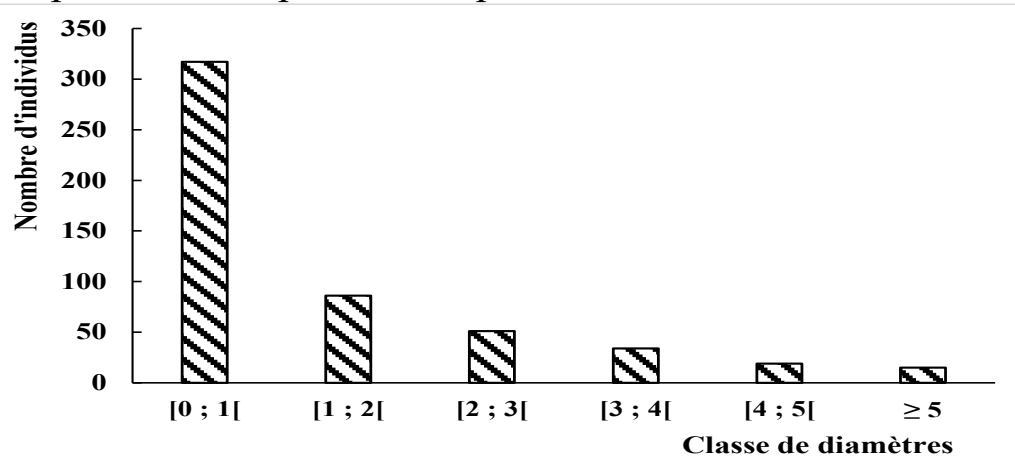

Figure 4: Répartition par classes de diamètres de l'ensemble des lianes de la Forêt Classée de Bouaflé 
Teck

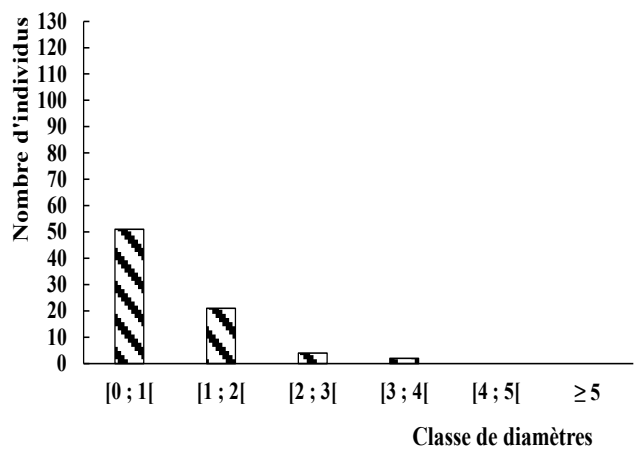

Jachère

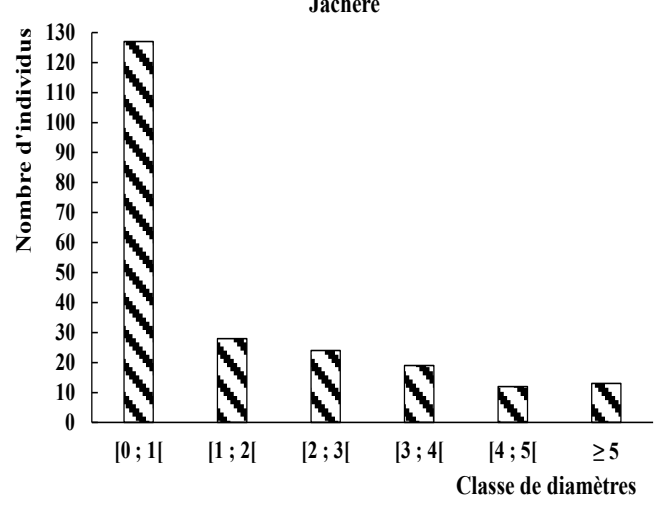

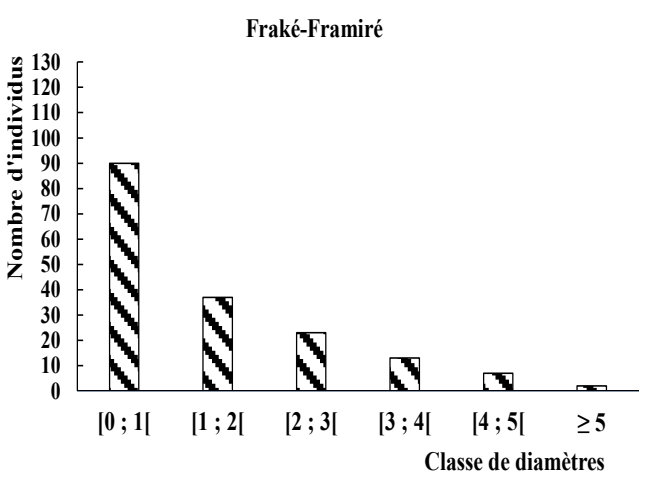

Hévéa

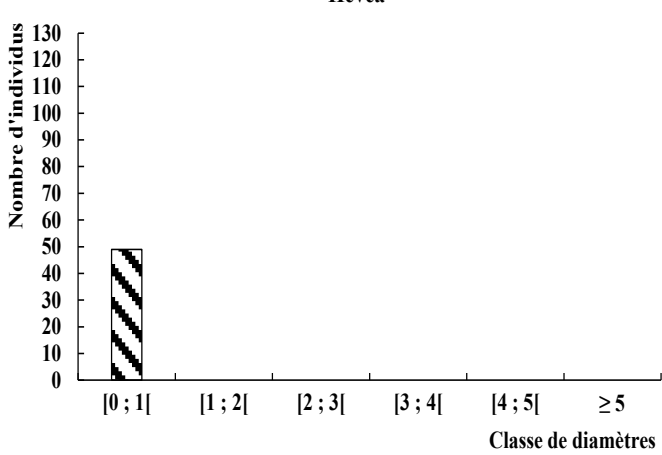

Figure 5: Structure diamétrique des lianes des différents types d'occupation du sol

\section{Discussion}

Cette étude a été conduite pour connaître la diversité des lianes dans la forêt classée de Bouaflé. Les inventaires réalisés dans quatre types d'occupation du sol (TOS) de cette forêt ont permis d'inventorier 522 individus de lianes. Ces lianes appartiennent à 45 espèces et sont reparties en 39 genres et 20 familles.

Ce résultat diffère de celui obtenu par Koffi et al. (2016) dans le parc national d'Azagny en Côte d'Ivoire. Ces auteurs ont recensé 5436 individus de lianes de diamètre à hauteur de poitrine $(\mathrm{DBH}) \geq 1 \mathrm{~cm}$ issues de 63 espèces reparties en 47 genres et 28 familles. Ce faible nombre obtenu malgré la considération de tous les DBH dans cette étude pourrait être dû d'une part, au fait que les deux milieux aient des types de végétation différents et d'autre part à la taille de l'échantillon inventorié et à la méthode d'étude utilisée. En effet, nous n'avons utilisé que la méthode de relevé de surface sur une surface réduite contrairement à ces auteurs qui ont utilisé une association de méthodes d'inventaire sur une surface plus grande. Or, l'association de plusieurs 
méthodes d'inventaire permet d'obtenir plus d'individus même dans des surfaces réduites (Vroh et al., 2010).

Toutefois, les nombres de familles (20) et genres (39) obtenus dans cette étude est supérieur à celui de Alemo (2014) dans la forêt mature de Yasikia en République Démocratique du Congo. Cet auteurn'a inventorié que 16 familles et 32 genres.

Adenia lobata (Passifloraceae) a été l'espèce la plus abondante. L'abondance de cette espèce a également été signalée par Ouattara (2008) et Koffi et al. (2016) respectivement dans la parcelle Henri Konan Bédié du jardin botanique de Bingerville et dans le Parc National d'Azagny au Sud de la Côte d'Ivoire. Ce résultat pourrait être due à la forte capacité de dissémination de la plante par les agents disséminateurs dont les insectes et les oiseaux et à une faible abondance de prédateurs de ses diaspores (Koffi et al., 2016). En effet, la dissémination par les animaux permet aux diaspores de franchir de grandes distances. Cela favorise l'extension de l'espèce et la diversification de son patrimoine génétique.

Cette étude a aussi montré que les Passifloraceae, les Fabaceae, les Caesalpiniaceae et les Combretaceae (avec 257 individus au total) ont été les familles les plus importantes dans la forêt classée de Bouaflé. Les Fabaceae font aussi partie des familles les plus importantes obtenues par Koffi et al. (2016) dans le Parc National d'Azagny en Côte d'Ivoire et par Gnahoré et al. (2020) à la périphérie du Parc National du Banco (Côte d'Ivoire).

En comparant les TOS, les familles des Passifloraceae et des Fabaceae ont été les seules présentes parmi les familles importantes dans trois TOS. Par contre, les Passifloraceae ont été absentes dans les teckeraies. L'absence des Passifloraceae dans ce TOS pourrait être due à l'ombrage engendrée par les feuilles de Teck. En effet, les espèces inventoriées appartenant à cette famille sont majoritairement des espèces héliophiles. Adenia lobata (Jacq.) Engl., par exemple, qui est, d'ailleurs, la plus abondante de cette famille dans notre étude est une espèce considérée comme exigeante en lumière (Koffi et al., 2016). La fermeture de la canopée et l'ombrage auraient empêché son développement ainsi que de celui de certaines autres espèces héliophiles de cette famille. L'importance des Passifloraceae dans les plantations d'hévéa peut s'expliquer par le fait que les espèces de cette famille se régénèrent plus rapidement que celles des autres. En effet, après le désherbage, ces espèces de cette famille seraient peut-être les premières à recoloniser le milieu.

La faible similarité floristique obtenue entre les plantations d'hévéa et les trois autres TOS indique que la flore lianescente des plantations d'Hévéa n'a pas de ressemblance significative avec celle des autres TOS. La majorité des lianes présentes dans les plantations de Teck, de Fraké-Framiré et dans les jachères serait donc différente de celles présentes dans les plantations d'hévéa. 
Cette différence pourrait être due à l'entretient (nettoyage) régulier dont bénéficie les plantations d'hévéa.

Dans l'ensemble des sites inventoriés, les individus jeunes (classe $[0 ; 1[$ ) ont été plus nombreux que les adultes (les autres classes). Cette abondance des individus jeunes par rapport aux adultes au niveau des jachères et des plantations de Fraké-Framiré pourrait s'expliquer par l'évolution naturelle des jachères ainsi que par la spontanéité des deux essences de Terminalia en Côte d'Ivoire. Ceci témoigne également d'une régénération naturelle de la flore au niveau de ces deux TOS (Angoni et al., 2018). Dans les conditions de développement optimales, sans perturbations ces jeunes lianes pourraient donc devenir de grosses lianes et contribuer ainsi à la reconstitution d'une forêt primaire.

La répartition des individus en classes de diamètres a montré une représentation en forme de $\mathrm{J}$ inversée. Ce type de forme est caractéristique des peuplements multi-spécifiques avec prédominance des individus jeunes d'où un fort potentiel de régénération (Kouyaté et al., 2020). Ce résultat montre que la flore lianescente de tous les milieux étudiés de la FCB est stable. Une telle représentation est caractéristique d'une forêt en bon état de conservation et à bonne capacité de régénération (Gueulou et al., 2018). Un résultat similaire a été obtenu par Alemo (2014) dans la forêt mature de Yasikia en République Démocratique du Congo. Toutefois dans les plantations de Teck et d'hévéa, toutes les classes de diamètre n'ont pas été représentées. Cela pourrait s'expliquer par la densité élevé (Teck) et l'entretien régulier des plantations d'hévéa et aussi par le fait que ces deux espèces soient des espèces introduites.

\section{Conclusion}

Cette étude a été réalisée pour connaître la diversité des lianes dans la forêt classée de Bouaflé. Pour ce faire, les inventaires floristiques basés sur les relevés de surface ont été réalisés dans quatre types d'occupation du sol (TOS). $\mathrm{Au}$ total, les inventaires floristiques réalisés dans quatre TOS ont permis d'inventorier 522 individus de 45 espèces de lianes reparties en 39 genres et 20 familles. Du point de vue floristique, les jachères ont été les milieux les plus riches avec 35 espèces repartis en 32 genres et 17 familles suivies des plantations de Fraké-Framiré avec 27 espèces reparties en 26 genres et 16 familles. Les plantations d'hévéa ont été le TOS le plus pauvres en lianes avec seulement 49 individus pour 11 familles, 15 genres et 16 espèces.

Dans l'ensemble des TOS, quatre familles botaniques ont été les plus importantes. Il s'agit des Passifloraceae, des Fabaceae, des Caesalpiniaceae et des Combretaceae. Parmi ces familles les Passifloraceae ont été communes aux TOS Fraké-Framiré, Jachère et Hévéa. Trois espèces ont été les plus importantes dans l'ensemble des TOS. Ce sont Adenia lobata (Jacq.) Engl., Acacia pennata (L.) Willd et Combretum zenkeri Engl. \& Diels. 
Quant aux individus inventoriés, ils ont présenté une structure diamétrique en $J$ inversé dans l'ensemble des TOS comme dans chaque TOS avec une dominance des individus de petit diamètre. Ces milieux sont viables et capables de se régénérer sans pression anthropique.

Ainsi, dans une politique de reboisement des forêts, il faudra privilégier les reboisements plurispécifiques aux monospécifiques afin de favoriser une diversification des espèces, plus particulièrement les lianes.

\section{Remerciements}

Les auteurs remercient la Société de Développement des Forêts (SODEFOR) pour l'accès à la forêt classée de Bouaflé.

\section{Conflit d'intérêt}

Les auteurs de cet article déclarent qu'ils n'existent aucun conflit d'intérêt. 
Annexe : Liste complète des espèces de liane inventoriées dans la forêt classée de Bouaflé

\begin{tabular}{|c|c|c|c|c|}
\hline $\mathrm{N}^{\circ}$ & Espèce & Famille & Type biologique & Chorologie \\
\hline 1 & Abrus precatorius Linn & Fabaceae & Lmp & GC-SZ \\
\hline 2 & Acacia pennata (L.) Willd & Fabaceae & $\mathrm{LmP}$ & $\mathrm{GC}$ \\
\hline 3 & Adenia cissampeloides (Planch. ex Hook.) Harms & Passifloraceae & Lmp & GC \\
\hline 4 & Adenia lobata (Jacq.) Engl. & Passifloraceae & Lmp & GC \\
\hline 5 & Alchornea cordifolia (Schum. \& Thonn.) Müll.Arg. & Euphorbiaceae & Lmp & GC-SZ \\
\hline 6 & Argocoffeopsis afzelii (Hiern) Robbr. & Rubiaceae & Lmp & GC \\
\hline 7 & Caesalpinia bonduc (Linn.) Roxb & Caesalpiniaceae & Lmp & GC \\
\hline 8 & Calopogonium mucunoides Desv. & Fabaceae & Lmp & $\mathrm{GC}$ \\
\hline 9 & Cardiospermum grandiflorum $\mathrm{Sw}$. & Sapindaceae & Lmp & $\mathrm{GC}$ \\
\hline 10 & Centrosema pubescens Benth. & Fabaceae & Lmp & GC \\
\hline 11 & Cissus polyantha Gilg \& M. Brandt & Vitaceae & Lmp & GC \\
\hline 12 & Cnestis ferruginea DC & Connaraceae & Lmp & GC \\
\hline 13 & Combretum paniculatum Vent & Combretaceae & Lmp & GC-SZ \\
\hline 14 & Combretum zenkeri Engl. \& Diels & Combretaceae & Lmp & $\mathrm{GC}$ \\
\hline 15 & Cryptolepis calophylla (Baill.) L. Joubert \& Bruyns & Apocynaceae & Lmp & GC \\
\hline 16 & Dalbergia saxatilis Hook.f. & Fabaceae & Lmp & $\mathrm{GC}$ \\
\hline 17 & Dalechampia ipomoeifolia Benth. & Euphorbiaceae & Lmp & $\mathrm{GC}$ \\
\hline 18 & Griffonia simplicifolia (Vahl ex DC.) Bail & Caesalpiniaceae & Lmp & $\mathrm{GC}$ \\
\hline 19 & Icacina mannii Oliv. & Icacinaceae & Lmp & $\mathrm{GC}$ \\
\hline 20 & Ipomoea involucrata P. Beauv. & Convolvulaceae & LTh & GC-SZ \\
\hline 21 & Leptoderris fasciculata (Benth.) Dunn & Fabaceae & Lmp & $\mathrm{GC}$ \\
\hline 22 & Loeseneriella ectypetala $\mathrm{N}$. Hallé & Hippocrateaceae & $\mathrm{LmP}$ & GC \\
\hline 23 & Manniophyton fulvum Müll. Arg. & Euphorbiaceae & Lmp & GC \\
\hline 24 & Mezoneurum benthamianum Baill. & Caesalpiniaceae & Lmp & GC \\
\hline 25 & Mikania cordata (Burm.f.) B.L. Robinson var. cordata & Asteraceae & Lmp & GC \\
\hline 26 & Mimosa invisa Mart. & Mimosaceae & Lnp & $\mathrm{i}$ \\
\hline 27 & Mimosa pudica Linn. & Mimosaceae & Lnp & GC \\
\hline 28 & Morinda morindoides (Baker) Milne-Redh. & Rubiaceae & Lmp & $\mathrm{GC}$ \\
\hline 29 & Motandra guineensis A. DC. & Apocynaceae & Lmp & GC-SZ \\
\hline 30 & Passiflora edulis Sims & Passifloraceae & Lmp & $\mathrm{i}$ \\
\hline 31 & Passiflora foetida Linn. & Passifloraceae & Lmp & $\mathrm{GC}$ \\
\hline 32 & Paullinia pinnata $\mathrm{L}$ & Sapindaceae & Lmp & GC-SZ \\
\hline 33 & Pergularia daemia (Forsk.) Chiov. & Asclepiadaceae & Lmp & $\mathrm{GC}$ \\
\hline 34 & Phyllanthus muellerianus (Kuntze) Exell & Euphorbiaceae & Lmp & GC-SZ \\
\hline 35 & Piper guineense Schum. \& Thonn. & Piperaceae & Lmp & $\mathrm{GC}$ \\
\hline 36 & Pueraria phaseoloides (Roxb.) Benth. & Fabaceae & Lmp & $\mathrm{GC}$ \\
\hline 37 & Rutidea smithii Hiern subsp. Smithii & Rubiaceae & Lmp & $\mathrm{GC}$ \\
\hline 38 & Salacia owabiensis Hoyle & Hippocrateaceae & Lmp & GC \\
\hline 39 & Secamone afzelii (Schultes) K. Schum & Asclepiadaceae & Lmp & GC \\
\hline 40 & Strophanthus gratus (Hook.) Franch & Apocynaceae & Lmp & $\mathrm{GC}$ \\
\hline 41 & Strophanthus sarmentosus DC. & Apocynaceae & $\mathrm{LmP}$ & GC-SZ \\
\hline 42 & Tetracera affinis Hutch. & Dilleniaceae & Lmp & $\mathrm{GCW}$ \\
\hline 43 & Tetracera alnifolia Willd. Subsp alnifolia & Dilleniaceae & Lmp & GC-SZ \\
\hline 44 & Tragia benthamii Bak. & Euphorbiaceae & Lnp & $\mathrm{GC}$ \\
\hline 45 & Uvaria afzelii Sc. Elliot & Annonaceae & Lmp & $\mathrm{GC}$ \\
\hline
\end{tabular}

Lmp : Liane Microphanérophyte; LmP : Liane Mésophanérophyte; Lnp : Liane

Nanophanérophyte; GC : taxon de la région Guinéo-Congolaise ; i : taxon introduit ; GCW : taxon endémique du bloc forestier Ouest africain ; SZ : taxon de la région SoudanoZambézienne.

\section{References :}

1. Adjiri O.A., Assoma T.V., Soro G., Aka N., Bengaly I. \& Soro N. (2020). Évaluation des aptitudes d'usages des eaux de sources naturelles dans l'agriculture à partir de techniques hydrochimiques : 
cas de celles des départements de Daloa et Zoukougbeu, Côte d'Ivoire. Afrique Science, 16(1) : 204-217.

2. Alemo M.A. (2014). Structure et composition floristique des lianes de la forêt a Gilbertiodendron dewevrei (de wild) leonard. De yasikia (opala, r.d.c). Mémoire en biologie, Université de Kisangani (République Démocratique du Congo), 49 p.

3. Angoni H., Ongolo R.S., Ngodo M.J.B. \& Ngo M.M.L. (2018). Composition floristique, structure et menaces de la végétation de la ligne côtière de la Réserve de Faune de Douala-Edéa. Int. J. Biol. Chem. Sci., 12(2): 915-926.

4. Bongers F., Parren M.P.E. \& Traoré D. (2005). Forest Climbing Plants of West Africa. Diversity, Ecology and Management, 273 p.

5. Cottam G. \& Curtis J.T. (1956). The use of distance measures in phytosociological sampling. Ecology, 37(3): 451-460.

6. Cronquist A. (1960). The divisions and classes of Plants. The Botanical Review, 26(4): 425-482.

7. Dro B. (2014). Abondance in situ et efficacité in vivo des plantes médicinales à haut potentiel anthelminthique des forêts non protégées du Sud de la Côte d'Ivoire. Thèse de Doctorat, Université Félix Houphouët-Boigny (Côte d'Ivoire), 210 p.

8. Eilu G. \& Bukenya-Zirabab R. (2004). Local use of climbing plants of budongo forest reserve, western uganda. Journal of Ethnobiology, 24 (2): 307-327.

9. Ewango C. (2010). The liana assemblage of a congolian rainforest. Diversity, Structure and Dynamics. $161 \mathrm{P}$.

10. FAO (2005). Global forest resources assessment. Progress towards sustainable forest management. FAO Forestry Paper Rome (Italy), 147.

11. Gueulou N., Ouattara N. D., Konan D., Gnahoré E., Missa K. \& Bakayoko A. (2018). Diversité floristique et structurale de la forêt galerie du Bandama dans la Réserve Scientifique de Lamto en Côte d'Ivoire. Afrique Science, 14(4) : 439-452.

12. Gnahoré E., Koné M., Soro Y., N'guessan Y.J. \& Bakayoko A. (2020). Effets de l'anthropisation sur la diversité floristique à la périphérie du Parc National du Banco, Abidjan, Côte d'Ivoire. Afrique Science, 16(4) : 167-180.

13. Koffi A.B., Kouamé D. \& Adou Yao C.Y. (2016). Structure and composition of the liana assemblage of Azagny National Park in the Southern Côte d'Ivoire. International Journal of Biodiversity and Conservation, 8(8): 206-215.

14. Koubouana F., Ifo S. A., Ndzai S. F., Stoffenne B. \& MondzaliLénguya R. (2018). Étude comparative d'une forêt primaire et d'une 
forêt dégradée au Nord de la République du Congo par référence à la structure des forêts tropicales humides. Revue Scientifque et Technique Forêt et Environnement du Bassin du Congo, 11 :11-25.

15. Kouyaté A. M., Diarra I. \& Habou R. (2020). Composition floristique, diversité et structure des espèces forestières alimentaires de la région de Sikasso au Sud du Mali. European Scientific Journal, April 2020 édition vol.16.

16. Lebrun J.P. \& Stork A.L. (1991). Enumération des plantes à fleurs d'Afrique tropicale et Tropical African Flowering Plants: Ecology and Distribution. Conservatoire et Jardin botaniques de la Ville de Genève, vol. 1-7.

17. Lebrun J.P. \& Stork A.L. (2015). Enumération des plantes à fleurs d'Afrique tropicale et Tropical African Flowering Plants: Ecology and Distribution. Conservatoire et Jardin botaniques de la Ville de Genève, vol. 1-7.

18. Mori S.A., Boom B.M., Carvalino De A.M. \& Dos Santos T.S. (1983). Southern Bahia moist forest. Bot. Rev., 49(2) : 155-232.

19. Ouattara K.M. (2008). Inventaire floristique des espèces arburstives, lianescentes et herbacées de la parcelle Henri Konan Bédié du jardin botanique de Bingerville en Côte d'Ivoire. Mémoire de Maitrise, Université d'Abobo-Adjamé, Abidjan, Côte d'Ivoire. https://www.memoireonline.com/11/13/7732/m_Inventairefloristiques-des-espèces-arburstives-lianescentes-et-herbacees-de-laparcelle-Henri-kona.html, page consultée le 03/05/2019.

20. Schnitzer S.A. \& Bongers, F. (2002). The ecology of lianas and their role in forests. Trends in Ecology and Evolution, 17 (5): 223-230.

21. Sidibé O. (2015). Impacts des pressions anthropiques sur la diversité végétale de la forêt classée de Bouaflé (Centre-Ouest de la Côte d'Ivoire). Mémoire de Master, Université Jean Lorougnon Guédé, Daloa, Côte d'Ivoire, $75 \mathrm{p}$.

22. SODEFOR (2014). Plan d'aménagement de la forêt classée de Bouaflé. Ministère des Eaux et Forêts, Centre de Gestion de Daloa, Côte d'Ivoire, $76 \mathrm{p}$.

23. Sørensen T. (1948). A method of establishing group of equal amplitude in plant sociology based on similarity of species content and its application to analyse of the vegetation on Danish common. Kjöbenhavn, 5 (4) : 1-34.

24. Tra Bi F.H. (2002). Quelques lianes de la pharmacopée ivoirienne. La base de ressources documentaires de l'IRD. Publications des scientifiques de l'IRD, 449-452.

25. Vroh B.T.A., Adou Yao C.Y., Kouamé D., N'Da D.H. \& N'Guessan K.E. (2010). Diversités Floristique et Structurale sur le Site d'une 
Réserve Naturelle Volontaire à Azaguié, Sud-est de la Côte d'Ivoire. European Journal of Scientific Research 45 (3): 411-421.

26. Yao F.E. (2017). Impact des types de reboisement sur la conservation de la diversité végétale dans la forêt classée de Bouaflé (Centre-Ouest de la Côte d'Ivoire). Mémoire de Master, Université Jean Lorougnon Guédé, Daloa, Côte d'Ivoire, 61 p. 